\title{
Late Gadolinium Enhancement in Cardiac Magnetic Resonance Imaging Is Associated with High Renal Resistive Index in Patients with Systemic Sclerosis
}

\author{
Rosario Cianci $^{a} \quad$ Antonietta Gigante ${ }^{a}$ Maria Ludovica Gasperini ${ }^{a}$ \\ Biagio Barbano $^{a}$ Nicola Galea $^{b}$ Edoardo Rosato ${ }^{a}$ \\ aDepartment of Translational and Precision Medicine, Sapienza University of Rome, Rome, \\ Italy; ${ }^{b}$ Department of Radiological, Oncological and Pathological Sciences, Sapienza \\ University of Rome, Rome, Italy
}

\section{Keywords}

Systemic sclerosis · Renal resistive index · Renal Doppler ultrasound · Late gadolinium enhancement $\cdot$ Cardiac magnetic resonance

\begin{abstract}
Introduction: Systemic sclerosis (SSc) is a chronic autoimmune disease characterized by microvascular damage and fibrosis of the skin and internal organs. The major complications are lung fibrosis, pulmonary artery hypertension, scleroderma renal crisis, and cardiac involvement. Objective: The aim of this study was to assess renal and cardiac involvement in asymptomatic SSc patients using renal Doppler ultrasound (RDU) and cardiac magnetic resonance (CMR). Materials and Methods: We enrolled 26 consecutive SSc patients ( 21 female) according to 2013 ACR/EULAR criteria. Biochemical analysis, clinical evaluation, RDU with intrarenal hemodynamic parameters (renal resistive index [RRI], pulsatility index [PI], systolic/diastolic [S/D] ratio), and CMR with late gadolinium enhancement (LGE) were investigated at the time of enrollment. Results: The median PI value was significantly $(p=0.007)$ higher in SSc patients with LGE than in SSc patients without LGE (1.37 [1.28-1.58] vs. 1.12 [1.06-1.26]). The median RRI value was significantly $(p=0.002)$ higher in SSc patients with LGE than in SSc patients without LGE $(0.68[0.65-0.73]$ vs. 0.64 [0.63-0.65]). The median S/D ratio was significantly ( $p=$ $0.02)$ higher in SSc patients with LGE than in SSc patients without LGE (3.12 [2.83-3.76] vs. 2.78 [2.64-2.84]). Conclusions: Our study, although performed on a small SSc population, showed RRI and LGE as markers of vascular and fibrotic damage. Early detection of cardiorenal involvement in SSc patients without symptoms is important to avoid further complications.
\end{abstract}




\section{Kidney \\ Blood Pressure \\ Research}

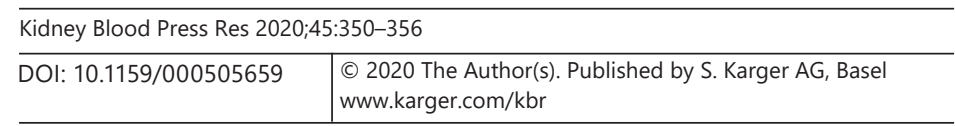

Cianci et al.: Systemic Sclerosis and Intrarenal Arterial Stiffness

\section{Introduction}

Systemic sclerosis (SSc) is an autoimmune disease characterized by endothelial dysfunction and fibrosis of the skin and internal organs [1]. The hallmark of the disease is Raynaud phenomenon (RP) that manifests as vasospastic attack generating chronic hypoxia. Internal organ complications are common in SSc patients and often remain asymptomatic until the late stages of disease. The major complications are lung fibrosis, pulmonary artery hypertension (PAH), scleroderma renal crisis (SRC), and cardiac involvement. In the past, before the introduction of angiotensin-converting enzyme (ACE) inhibitors, kidney involvement was the most frequent cause of death due to SRC [2]. To date, renal complications due to SSc are frequently reported as reduced glomerular filtration rate (GFR), proteinuria, antiphospholipid-associated nephropathy, myeloperoxidase-antineutrophil cytoplasmic antibody-associated glomerulonephritis, and high intrarenal arterial stiffness [3]. Cardiac involvement is characterized by fibrosis, conduction system abnormalities, and myositis but is also associated with other organ complications, such as PAH and SRC [4]. The presence of renal and cardiac involvement in SSc is often underestimated depending on the methods used. The aim of the study was to assess renal and cardiac involvement in asymptomatic SSc patients using renal Doppler ultrasound (RDU) to evaluate intrarenal arterial stiffness and cardiac magnetic resonance (CMR) with late gadolinium enhancement (LGE).

\section{Materials and Methods}

We recruited consecutiveSScpatients admitted to the Scleroderma Unit of the Department of Translational and Precision Medicine according to 2013 ACR/EULAR criteria [5]. Disease subset according to LeRoy et al. [1] and modified Rodnan skin score [6] were also collected. Clinical assessment, biochemical analyses, RDU, and CMR were recorded in all patients at the time of enrollment. Patients with PAH, heart failure, cardiac arrhythmias and conduction disorders, interstitial lung disease, hypertension, diabetes mellitus, dyslipidemia, valvular heart diseases, coronary artery disease, chronic kidney injury, glomerulonephritis, abnormal urinalysis, hepatic or thyroid dysfunction, and anemia were excluded.

At the time of the study, all SSc patients were undergoing treatment with calcium channel blockers (nifedipine $30 \mathrm{mg} /$ day), and the therapy was discontinued $72 \mathrm{~h}$ before the CMR and RDU.

None of the patients were treated with immunosuppressive agents or corticosteroids at an equivalent dose of prednisone $\geq 10 \mathrm{mg} /$ day, $\beta$-blockers, antiarrhythmic drugs, ACE inhibitors, or angiotensin receptor antagonists.

The subjects' written consent was obtained according to the Declaration of Helsinki, and the study was conducted in agreement with the local ethics committee's directives.

\section{Cardiac Magnetic Resonance}

CMR imaging was performed with a 1.5-T unit (Magnetom Avanto; Siemens Healthcare $\mathrm{GmbH}$, Erlangen, Germany) using an 8-channel phased-array coil and vectocardiogram triggering. LGE imaging was performed between 10 and 15 min after the second bolus injection of contrast agent using a segmented T1-weighted phase-sensitive inversion recovery pulse.

\section{Renal Doppler Ultrasound}

Toshiba Aplio Ultrasound System SSA-790 equipped with a convex 3.5-MHz probe was used to obtain images for intrarenal parameters. In each patient, renal resistive index (RRI), pulsatility index (PI), and systolic/diastolic (S/D) ratio were measured on the lateral paren- 
Kidney

Blood Pressure

Research

Fig. 1. Renal Doppler ultrasound with velocity maximum, renal resistive index, pulsatility index, and systolic/diastolic ratio.

Table 1. Characteristics of the SSc patients

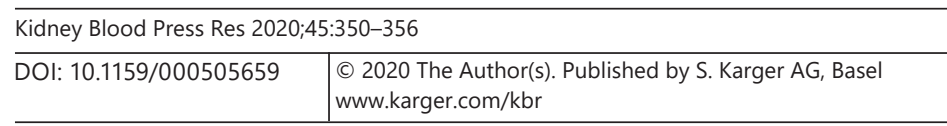

Cianci et al.: Systemic Sclerosis and Intrarenal Arterial Stiffness

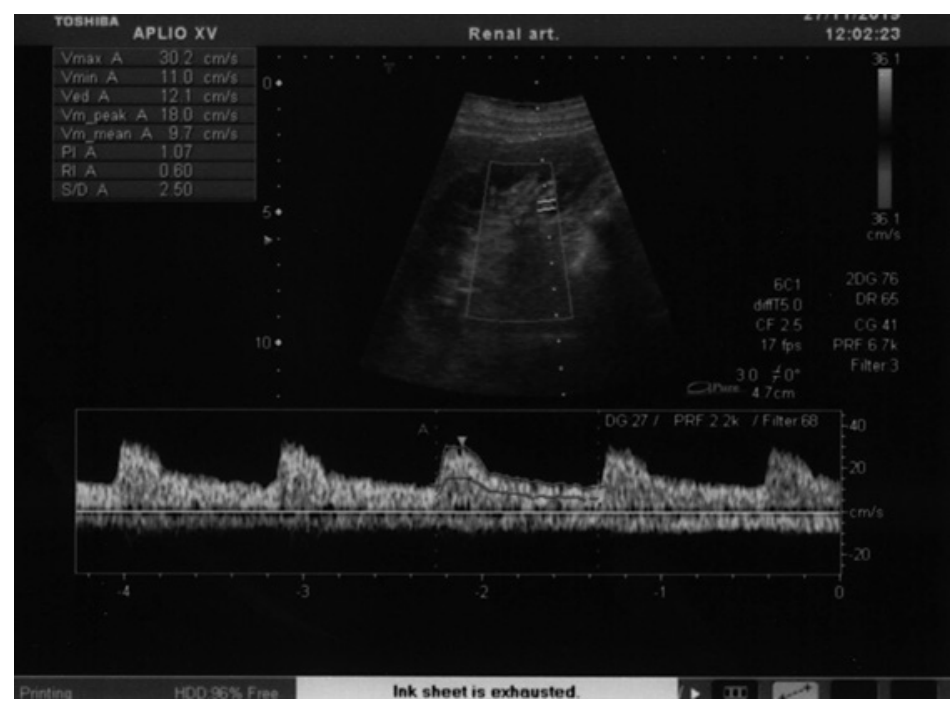

$\begin{array}{lc}\text { Patients, } n & 26 \\ \text { Age, years (median and CI) } & 42(38-47) \\ \text { Female, } n & 21 \\ \text { lcSSc/dcSSc } & 10 / 16 \\ \text { mRss (median and CI) } & 13(11-18) \\ \text { BMI, kg/m }{ }^{2} & 22 \pm 2.9 \\ \text { CKD-EPI, mL/min } & 103(89-111) \\ \text { Proteinuria, mg/24 h } & 98(76-143) \\ \text { NVC: early, } n(\%) & 6(23) \\ \text { NVC: active, } n(\%) & 10(38.5) \\ \text { NVC: late, } n(\%) & 10(38.5) \\ \text { DUs, } n(\%) & 16(61.5) \\ \text { PI (median and CI) } & 1.28(1.21-1.38) \\ \text { RRI (median and CI) } & 0.65(0.64-0.68) \\ \text { S/D (median and CI) } & 2.84(2.75-3.12) \\ \text { IVS, mm } & 8.8(8-9.2) \\ \text { LGE, } n(\%) & 16(61.5)\end{array}$

Values are medians (ranges) unless otherwise indicated. lcSSc, limited cutaneous SSc; dcSSc, diffuse cutaneous SSc; mRss, modified Rodnan skin score; BMI, body mass index; NVC, nailfold videocapillaroscopy; DUs, digital ulcers; PI, pulsatility index; RRI, renal resistive index; S/D, systolic/diastolic ratio; IVS, interventricular septum thickness; LGE, late gadolinium enhancement.

chyma of the kidney, at the interlobar/interlobular arteries directed towards the probe without angle correction [7].

RRI was measured as (peak systolic velocity - diastolic velocity)/peak systolic velocity. PI was calculated as (peak systolic frequency shift - minimum diastolic frequency shift)/ mean frequency shift (Fig. 1).

\section{Statistical Analysis}

The results were expressed as medians and ranges. Commercial software (SPSS version 25.0) was used for statistical analysis. The coefficient of kurtosis was used to evaluate the 
Fig. 2. Correlation between renal resistive index (RRI) and maximum velocity.

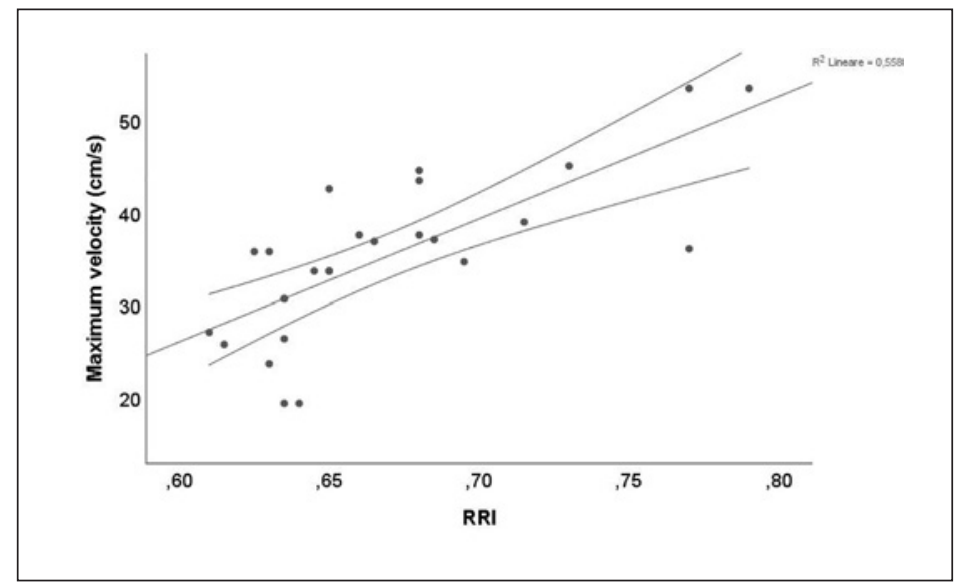

normal distribution of data. Group comparisons were made by Kruskal-Wallis test. Fisher's exact test was used to compare categorical variables. $p$ values $<0.05$ were considered significant.

\section{Results}

We included 26 SSc patients of whom 21 (81\%) were female. The clinical characteristics are shown in Table 1. Sixteen (61.5\%) patients had diffuse cutaneous SSc and 10 (38.5\%) had limited cutaneous SSc. The median value of interventricular septum thickness was 8.8 (8-9.2) $\mathrm{mm}$. No statistically significant difference $(p<0.05)$ regarding interventricular septum thickness was observed between SSc patients with and without LGE (8.5 [8-10] vs. 9 [9-10] $\mathrm{mm}$ ). LGE was present in 16 (61.5\%) SSc patients. The median value of eGFR was 103 (89-11) $\mathrm{mL} / \mathrm{min}$. The median value of proteinuria was 98 (76-143) mg/24 h. No statistically significant difference $(p<0.05)$ in proteinuria was observed between SSc patients with and without LGE (95 [8-150] vs. 99 [80-141] mg/24 h). The median values of intrarenal stiffness parameters were the following: maximum velocity 35.7 (33.6-37.5) cm/s, RRI 0.65 (0.64-0.68), PI 1.28 (1.21-1.38), and S/D ratio 2.84 (2.75-3.12). Figure 2 shows the correlation between RRI and maximum velocity. The median value of PI was significantly ( $p=0.007)$ higher in SSc patients with LGE than in SSc patients without LGE (1.37 [1.28-1.58] vs. 1.12 [1.06-1.26]). The median value of RRI was significantly $(p=0.002)$ higher in SSc patients with LGE than in SSc patients without LGE (0.68 [0.65-0.73] vs. 0.64 [0.63-0.65]). The median value of the S/D ratio was significantly ( $p=0.02$ ) higher in SSc patients with LGE than in SSc patients without LGE (3.12 [2.83-3.76] vs. 2.78 [2.64-2.84]) (Fig. 3). Table 2 shows the differences between SS patients with and without LGE and renal Doppler findings.

\section{Discussion}

Our data showed that intrarenal parameters and LGE could be diagnostic markers of fibrosis in asymptomatic SSc patients. It is well known that renal scleroderma-associated vasculopathy is characterized by abnormal RRI [8] and endothelial markers [9]. In the absence of SRC or other acute conditions, such as glomerulonephritis and associated vasculitis, the vascular renal involvement in SSc has a benign course [3] and correlates with GFR, digital 


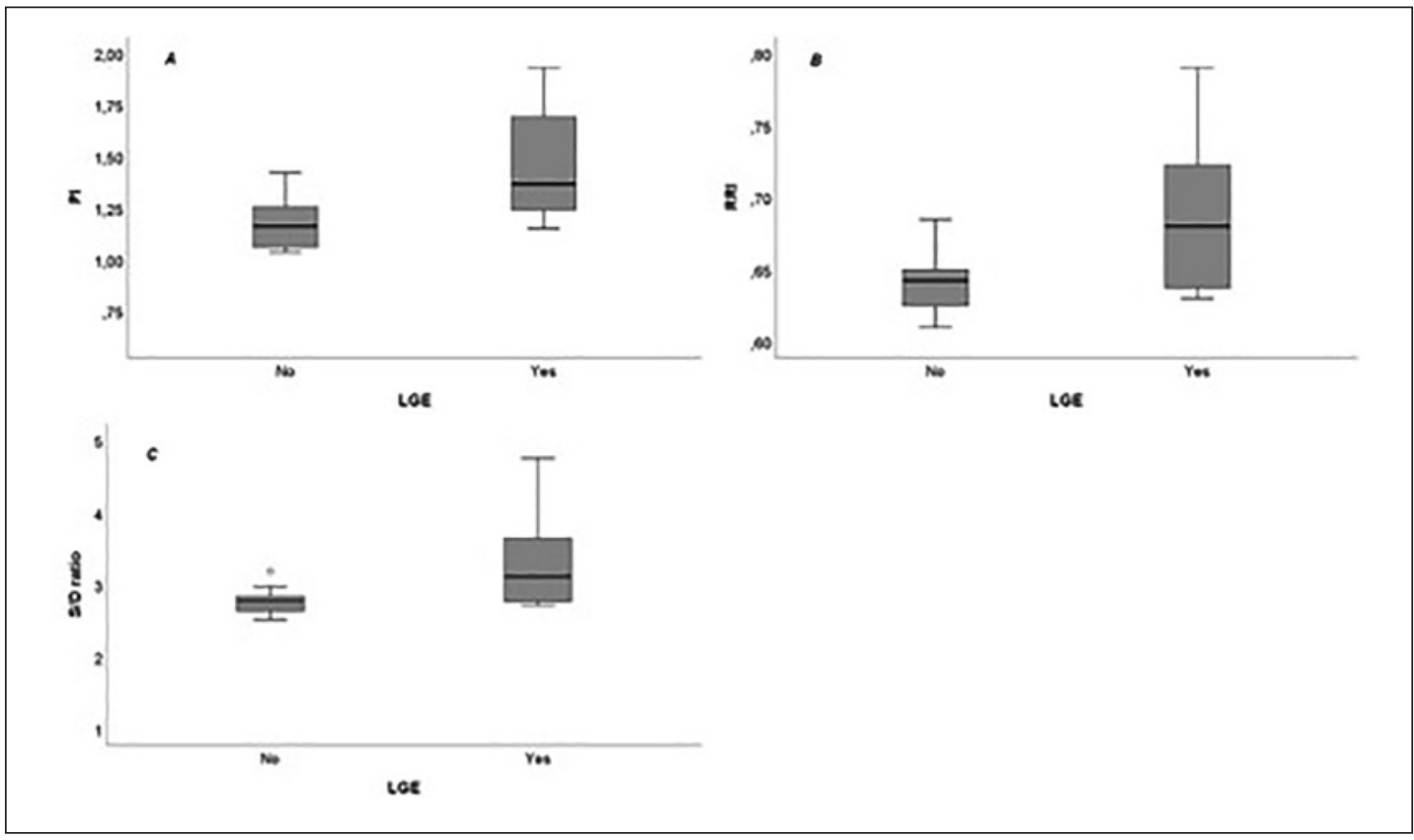

Fig. 3. The median value and confidence interval of pulsatility index (PI) (a), renal resistive index (RRI) (b), and systolic/diastolic (S/D) ratio (c) in SSc patients with late gadolinium enhancement (LGE) and without LGE.

Table 2. Differences between SSc patients with and without LGE and renal Doppler findings

\begin{tabular}{llll}
\hline & LGE & No LGE & $p$ value \\
\hline RRI (median and CI) & $0.68(0.65-0.73)$ & $0.64(0.63-0.65)$ & 0.002 \\
PI (median and CI) & $1.37(1.28-1.58$ & $1.12(1.06-1.26)$ & 0.007 \\
S/D (median and CI) & $3.11(2.84-3.76)$ & $2.78(2.64-2.84)$ & 0.02 \\
\hline
\end{tabular}

LGE, late gadolinium enhancement; RRI, renal resistive index; PI, pulsatility index; S/D, systolic/diastolic ratio.

ulcers [10], and impaired autonomic activity [11]. In scleroderma, RRI is a useful marker to evaluate endothelial dysfunction in several damaged organs [12-14].

Bruni et al. [7], in a large cohort of 380 SSc patients, showed that RRI was an independent risk factor for death in association with skin involvement, lung fibrosis, dyspnea, and late scleroderma pattern. Thus, they concluded that RRI appears to be a potential marker of fibrosis both for renal and systemic involvement. Several studies have been conducted in renal tissues from autopsies, finding vascular involvement with increased intimal thickening in SSc patients compared to healthy controls, while luminal occlusion was observed in patients with the diffuse cutaneous subset of the disease and SRC. Furthermore, nonocclusive fibrosis in small renal arteries was observed in SSc patients without SRC [15].

In the past, left ventricular mass and RRI have been reported as early diagnostic markers in cardiorenal syndrome type 5 due to scleroderma [16]. Although SSc is a systemic disease, cardiac involvement is often underestimated due to the methods used. Also, some symptoms related to cardiac complications are often attributed to pulmonary or esophageal involvement 
[4]. Myocardial fibrosis has been reported in $>50 \%$ of autopsy studies representing the pathological hallmark. CMR with LGE is considered a noninvasive tool in detecting myocardial fibrosis. Gargani et al. [17], in a large cohort of SSc patients, found early cardiac involvement using CMR. LGE was detected in 56 of 201 patients and correlated with ventricular arrhythmias.

$\mathrm{RP}$ is the hallmark of SSc, promoting chronic hypoxia, microvascular dysfunction with collagen deposition in the tissue, and consequent fibrosis [18]. In patients with SSc, the hypoxia secondary to RP vasospasm enhances renal and systemic vasoconstriction, increasing intrarenal hemodynamic parameters, such as RRI, PI, and S/D, and reducing renal longitudinal diameter and parenchymal thickness. Subsequently, interstitial fibrosis and cortical microcirculation dysfunction lead to glomerulosclerosis [19]. Several studies have already demonstrated that RRI represents a systemic marker of vascular damage and fibrosis [7, 20] and is a predictor of cardiovascular risk [21,22]. Except for SRC, kidney involvement in SSc is subclinical and is associated with vascular damage. Since high RRI is related to vascular involvement, further assessments are needed to better evaluate complications due to the disease. In SSc, a pathological RRI showed a negative impact on mortality, and for this reason, it should be taken into consideration in association with other systemic complications, such as cardiac involvement.

\section{Conclusions}

Our study, although performed on a small SSc population, showed an association between RRI and LGE. CMR with LGE and RRI are noninvasive, nonradiating methods to timely detect cardiorenal involvement in SSc, and it could be useful to add them in the routine evaluation. Early detection of pathological findings in asymptomatic SSc patients is important to avoid further complications and allows specific therapy.

\section{Statement of Ethics}

All procedures performed in studies involving human participants were in accordance with the ethical standards of the institutional and/or national research committee. Informed consent was obtained from all individual participants included in the study.

\section{Disclosure Statement}

The authors declare that no conflict of interest exists.

\section{Funding Sources}

This study was not funded.

\section{Author Contributions}

The authors are responsible for the content and writing of the paper. The manuscript has been seen and approved by all authors. 


\section{Kidney \\ Blood Pressure \\ Research}

\begin{tabular}{l|l}
\hline Kidney Blood Press Res 2020:45:350-356 \\
\hline DOI: 10.1159/000505659 & $\begin{array}{l}\text { @ 2020 The Author(s). Published by S. Karger AG, Basel } \\
\text { www.karger.com/kbr }\end{array}$ \\
\hline
\end{tabular}

Cianci et al.: Systemic Sclerosis and Intrarenal Arterial Stiffness

\section{References}

1 LeRoy EC, Black C, Fleischmajer R, Jablonska S, Krieg T, Medsger TA Jr, et al. Scleroderma (systemic sclerosis): classification, subsets and pathogenesis. J Rheumatol. 1988 Feb;15(2):202-5.

2 Traub YM, Shapiro AP, Rodnan GP, Medsger TA, McDonald RH Jr, Steen VD, et al. Hypertension and renal failure (scleroderma renal crisis) in progressive systemic sclerosis. Review of a 25-year experience with 68 cases. Medicine (Baltimore). 1983 Nov;62(6):335-52.

3 Rosato E, Gigante A, Barbano B, Gasperini ML, Cianci R, Muscaritoli M. Prognostic Factors of Renal Involvement in Systemic Sclerosis. Kidney Blood Press Res. 2018;43(3):682-9.

4 Champion HC. The heart in scleroderma. Rheum Dis Clin North Am. 2008 Feb;34(1):181-90.

5 van den Hoogen F, Khanna D, Fransen J, Johnson SR, Baron M, Tyndall A, et al. 2013 classification criteria for systemic sclerosis: an American College of Rheumatology/European League against Rheumatism collaborative initiative. Arthritis Rheum. 2013 Nov;65(11):2737-47.

6 Clements P, Lachenbruch P, Siebold J, White B, Weiner S, Martin R, et al. Inter and intraobserver variability of total skin thickness score (modified Rodnan TSS) in systemic sclerosis. J Rheumatol. 1995 Jul;22(7):1281-5.

7 Bruni C, Rosato E, Maestripieri V, Gigante A, Tesei G, Bellando-Randone S, et al. The Renal Resistive Index in systemic sclerosis: Determinants, prognostic implication and proposal for specific age-adjusted cut-offs. Eur J Intern Med. 2019 Dec;70:43-49.

8 Shanmugam VK, Steen VD. Renal manifestations in scleroderma: evidence for subclinical renal disease as a marker of vasculopathy. Int J Rheumatol. 2010;2010:538589.

9 Gigante A, Navarini L, Margiotta D, Amoroso A, Barbano B, Cianci R, et al. Angiogenic and angiostatic factors in renal scleroderma-associated vasculopathy. Microvasc Res. 2017 Nov;114:41-5.

10 Rosato E, Gigante A, Barbano B, Cianci R, Molinaro I, Rossi C, et al. Intrarenal hemodynamic parameters correlate with glomerular filtration rate and digital microvascular damage in patients with systemic sclerosis. Semin Arthritis Rheum. 2012 Jun;41(6):815-21.

11 Gigante A, Rosato E, Liberatori M, Barbano B, Cianci R, Gasperini ML, et al. Autonomic dysfunction in patients with systemic sclerosis: correlation with intrarenal arterial stiffness. Int J Cardiol. 2014 Dec;177(2):578-80.

12 Rosato E, Barbano B, Gigante A, Aversa A, Cianci R, Molinaro I, et al. Erectile dysfunction, endothelium dysfunction, and microvascular damage in patients with systemic sclerosis. J Sex Med. 2013 May; 10(5): 1380-8.

13 Rosato E, Gigante A, Barbano B, La Marra F, Molinaro I, Quarta S, et al. Clitoral blood flow in systemic sclerosis women: correlation with disease clinical variables and female sexual dysfunction. Rheumatology (Oxford). 2013 Dec;52(12):2238-42.

14 Rosato E, Gigante A, Barbano B, Cianci R, Molinaro I, Pisarri S, et al. In systemic sclerosis macrovascular damage of hands digital arteries correlates with microvascular damage. Microvasc Res. 2011 Nov;82(3): $410-5$.

15 Trostle DC, Bedetti CD, Steen VD, Al-Sabbagh MR, Zee B, Medsger TA Jr. Renal vascular histology and morphometry in systemic sclerosis. A case-control autopsy study. Arthritis Rheum. 1988 Mar;31(3):393-400.

16 Gigante A, Barilaro G, Barbano B, Romaniello A, Di Mario F, Quarta S, et al. Left Ventricular Mass and Intrarenal Arterial Stiffness as Early Diagnostic Markers in Cardiorenal Syndrome Type 5 due to Systemic Sclerosis. Cardiorenal Med. 2016 Feb;6(2):135-42.

17 Gargani L, Todiere G, Guiducci S, Bruni C, Pingitore A, De Marchi D, et al. Early Detection of Cardiac Involvement in Systemic Sclerosis: The Added Value of Magnetic Resonance Imaging. JACC Cardiovasc Imaging. 2019 May; 12(5):927-8.

18 Di Benedetto P, Ruscitti P, Liakouli V, Cipriani P, Giacomelli R. The Vessels Contribute to Fibrosis in Systemic Sclerosis. Isr Med Assoc J. 2019 Jul;21(7):471-4.

19 Gigante A, Barbano B, Gasperini ML, Zingaretti V, Cianci R, Rosato E. Renal Parenchymal Thickness in Patients with Systemic Sclerosis Is Related to Intrarenal Hemodynamic Variables and Raynaud Renal Phenomenon. J Rheumatol. 2019 Jun 15. pii: jrheum.190165.

20 Gigante A, Barbano B, Barilaro G, Quarta S, Gasperini ML, Di Mario F, et al. Serum uric acid as a marker of microvascular damage in systemic sclerosis patients. Microvasc Res. 2016 Jul;106:39-43.

21 Geraci G, Mulè G, Geraci C, Mogavero M, D'Ignoto F, Morreale M, et al. Association of renal resistive index with aortic pulse wave velocity in hypertensive patients. Eur J Prev Cardiol. 2015 Apr;22(4):415-22.

22 Mulè G, Geraci G, Geraci C, Morreale M, Cottone S. The renal resistive index: is it a misnomer? Intern Emerg Med. 2015 Dec;10(8):889-91. 